\title{
The Influence of Inhomogeneities on the Cardiac-Magnetic-Field Distribution (*).
}

\author{
M. J. Peters, M. J. M. Swennenhuts, A. vaN Oosterom \\ and J.J. WEVERS-HENKE \\ Twente University of Technology, Department of Technical Physics \\ P.O.B. 217, 7500 AE Enschede, The Netherlands
}

(ricevuto il 12 Novembre 1982)

Summary. - Numerical computations were performed on the magnetic field generated by the heart during ventricular depolarization. The purpose of this study was to investigate the contribution of inhomogeneities in the volume conductor to the total field and to establish the influence of gradiometers as used in experimental recordings.

PACS. 87.40. - Biomagnetism (including magnetocardiography).

PACS. 85.25. - Superconducting devices; superconducting magnets.

\section{1. - Introduction.}

One of the basic problems in magnetocardiography is to establish if and how recordings of the magnetic signals related to the electrical activity of the heart present just outside the body may assist in the diagnosis of cardiac malfunctioning. In this perspective it is worth considering if, or how, magnetocardiography can replace or complement standard electrocardiographic routines.

In earlier publications on magnetocardiography special attention has been given to the magnetic fields generated by current dipoles inside volume con-

(*) Paper presented at the "IV International Workshop on Biomagnetism ", held in Rome, September 14-16, 1982. 
ductors either of semi-infinite extent or bounded by a sphere ( ${ }^{1}$ ). It was expected from these studies that the magnetic fields would predominantly reflect the so-called primary sources within the heart and would be less affected by the secondary sources related to the volume currents inside the torso than electrocardiographic signals. In subsequent work the models have become more and more realistic in terms of both the cardiac generators and of the complexity of the volume conductor $\left(^{2,3}\right)$. A more complex picture related to the relative importance of the secondary sources evolved from these later studjes.

The observation points in these studies could not be realized during actual recordings of MCG data. Furthermore, no attention has been paid so far to the magnetic field at the posterior side of the torso. Hence we decided to set up a similar study which, in addition, would enable us to evaluate the effect of a gradiometer which is commonly used in the recording of experimental data. Such gradiometers perform a spatial differentiation of the flux of the magnetic field and their output is only approximately the magnetic field. Their infuence can only be assessed when a model is used which incorporates, as fully as is technically possible, the complexity of both the heart as a generator and the body tissues as a volume conductor.

\section{2. - Theory.}

The magnetic field $\boldsymbol{B}$ in a point $\boldsymbol{r}$ outside a bounded, piecewise homogeneous, volume conductor can be computed from $\left({ }^{4}\right)$

$$
\boldsymbol{B}(\boldsymbol{r})=\boldsymbol{B}_{\infty}(\boldsymbol{r})-\frac{\mu_{0}}{4 \pi} \sum_{i} \Delta \sigma_{i} \int_{s_{i}} V_{i}\left(\boldsymbol{r}^{\prime}\right) \frac{\boldsymbol{r}-\boldsymbol{r}^{\prime}}{\left|\boldsymbol{r}-\boldsymbol{r}^{\prime}\right|^{3}} \times \mathrm{d} \boldsymbol{S}_{i}^{\prime}
$$

with

$$
\begin{aligned}
& S_{i} \text { the interfaces between the various compartments; } \\
& \sum_{i} \text { the summation over all interfaces, the outer surface included; } \\
& \Delta \sigma_{i} \text { the conductivity just inside } S_{i} \text { minus that just outside; } \\
& V_{i} \text { the electrical potential at interface } S_{i} ; \\
& \boldsymbol{r}^{\prime} \text { a source point at an interface. }
\end{aligned}
$$

In this formulation $\boldsymbol{B}_{\omega}(\boldsymbol{r})$ is the field that arises from the primary sources, whereas the remainder reflects the effect of the secondary sources which are

(1) B. N. Cuffin and D. Cohen: IEEE Trans. Biomed. Eng., BME-24, 4 (1977).

$\left({ }^{2}\right)$ B. M. HoRACEK: IEEE Trans. Magn., MAG-9, 3 (1973).

$\left.{ }^{3}\right)$ B. N. Cuffin: IEEE Trans. Biomed. Eng., BME-28, 11 (1981).

(4) D. B. Geselowitz: IEEE Trans. Magn., MAG-6, 2 (1970). 
located at the interfaces $S_{i}$ of the various compartments of the inhomogeneous volume conductor.

The description of the primary sources that we used is the classical description of the cardiac electrical generator during depolarization, i.e. the uniform current dipole layer situated in a volume conductor of infinite extent. This is a surface which at any time instant during depolarization separates the cells of the cardiac muscle which have been depolarized and those which are still at rest. It can be shown $\left(^{5}\right)$ that for this source description the magnetic field $\boldsymbol{B}_{\infty}(\boldsymbol{r})$ at each time instant reads

$$
\boldsymbol{B}_{\infty}(\boldsymbol{r})=\frac{\mu_{0} \tau}{4 \pi} \oint \frac{\mathrm{d} \boldsymbol{l}^{\prime}}{\left|\boldsymbol{r}-\boldsymbol{r}^{\prime}\right|},
$$

where

$d \boldsymbol{l}^{\prime}$ is an infinitesimal element of the rim of the dipole layer, situated on the ventricular surface (both endocardium and epicardium);

$\tau$ is the current dipole density of the layer (unit $\mathbf{A} \cdot \mathbf{m}^{-1}$ ).

The contribution to the magnetic field of the secondary sources follows from an integration of the weighted potential over the interfaces. Hence these potential values must be known and can be obtained from the following integral equation $(6,7)$ :

$$
V_{k}(\boldsymbol{r})=\frac{\sigma}{\sigma_{k}} V_{\infty}(r)-\frac{1}{4 \pi \sigma_{k}} \sum_{i} \Delta \sigma_{i} \int_{s_{i}} V_{i}\left(\boldsymbol{r}^{\prime}\right) \frac{\left(\boldsymbol{r}-\boldsymbol{r}^{\prime}\right)}{\left|\boldsymbol{r}-\boldsymbol{r}^{\prime}\right|^{3}} \cdot d \boldsymbol{S}_{i}^{\prime}
$$

with

$\sigma_{k}$ the average of the electrical conductivities at both sides of boundary $\mathbb{S}_{k}$,

$\sigma$ the conductivity of the source region (the ventricular wail).

In this formulation $V_{\infty}(\boldsymbol{r})$ is the potential that arises from the primary sources, whereas the remainder reflects the effect of the secondary sources.

For a uniform current dipole layer with dipole density $\tau=\sigma V_{d}$, where $V_{d}$ is the potential drop over the dipole layer, the term $V_{\infty}(r)$ can be equated as

$$
V_{\infty}(\boldsymbol{r})=\frac{1}{4 \pi \sigma} \tau \Omega=V_{\mathrm{d}} \frac{\Omega}{4 \pi},
$$

with $\Omega$ the solid angle subtended by the layer at the observation point $r$.

${ }^{5}$ S) Rush: IEEE Trans. Biomed. Eng., BME-22, 3 (1975).

$\left({ }^{6}\right)$ A. C. L. Barnard, I. M. DUCK, M. S. LYNN and W. P. TIMLAKe: Biophys. J., 7, 476 (1967).

(7) R. C. Barr, T. C. Pilkington, J. P. Boineau and M. S. Spach: IEeE Trans. Biomed. Eng., BME-13, 88 (1966). 
Expressions (1)-(4) form the basis for our computations. Computing the total magnetic flux through the loops of a gradiometer leads to the evaluation of a $B_{\text {eff }}$ being the total flux through the gradiometer divided by the area of the pick-up loop.

\section{3. - Methods.}

The activation data from a study by DuRRer et al. $\left.{ }^{8}\right)$, who measured the depolarization sequence of a revived, perfused human heart, were used in the following way: on the closed surface $S_{\mathrm{h}}$ bounding the ventricular mass (endocardium and epicardium) a total of 283 points was distributed evenly and the reported depolarization time instants were assigned to them. These points formed the vertices of a total of 562 small plane triangles approximating $S_{h}$ : The rims on $S_{\mathrm{h}}$ of the dipole layers were computed by means of linear inter-

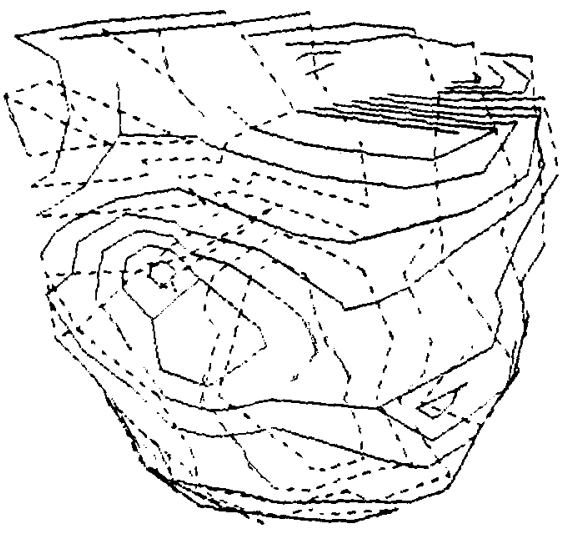

a)

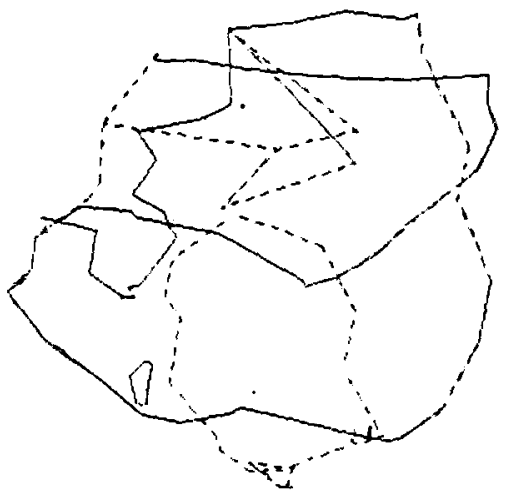

b)

Fig. 1. - a) The isochronic lines on the endocardium and epicardium of the ventricles, displayed $5 \mathrm{~ms}$ apart. b) The isochronic lines at $40 \mathrm{~ms}$ after onset of depolarization, isolated from $a$ ).

polation over each triangle for 11 consecutive time instants of $5 \mathrm{~ms}$ interval. These rims form a complex contour in space. In fig. 1a) a frontal view of $S_{h}$ is given where isochronic lines (rims) are displayed $5 \mathrm{~ms}$ apart. Figure $1 b$ ) shows a projection of the rim at $40 \mathrm{~ms}$. The rims were used for the computation of the primary sources of the magnetic field according to eq. (1). Those same rims determine the value of the solid angle as required in the source term of

$\left(^{8}\right)$ D. Durrer, R. T. van Dam, G. E. Freud, M. J. Janse, F. L. Meyler and R. C. Aktzbateher: Circulation, 41, 899 (1970). 
the electrical potential (eq. (4)). The value of $V_{\mathrm{d}}$ was taken as $40 \mathrm{mV}$; the value of $\sigma$ as $0.2 \mathrm{mho} \mathrm{m}^{-1}\left({ }^{9}\right)$.

The volume conductor consisted of a realistically shaped torso in which four compartments of different conductivity were placed, representing the two lungs and the two intercavitary blood masses. The geometry of the lungs was read from a standard anatomical reference $\left({ }^{10}\right)$, the shape of the intercavitary blood masses was taken in agreement with the heart data used. The integral equation (3) was solved numerically according to the method of Barr et al. (11) which is a variation of the method of Barnard et al. $\left(^{6}\right)$ in which the potential is computed at the vertex points of triangulated versions of the surfaces considered, rather than at the centres of these triangles. The triangulation of the torso shape has been described previously $\left({ }^{12}\right)$.

The resulting piecewise homogeneoues volume conductor is specified in table I.

The co-ordinates of the vertex points were read into a digital-computer

\begin{tabular}{llll}
\hline Surface & $\begin{array}{l}\text { Number of } \\
\text { vertex points }\end{array}$ & $\begin{array}{l}\text { Number of } \\
\text { triangles }\end{array}$ & $\begin{array}{l}\text { Conductivity } \\
\text { in mho } \mathrm{m}^{-1}\end{array}$ \\
\hline torso & 194 & 384 & 0.2 \\
\hline right lung & 101 & 198 & 0.04 \\
\hline left lung & 107 & 210 & 0.04 \\
\hline right ventricular cavity & 82 & 160 & 0.6 \\
\hline left ventricular cavity & 90 & 176 & 0.6 \\
\hline
\end{tabular}

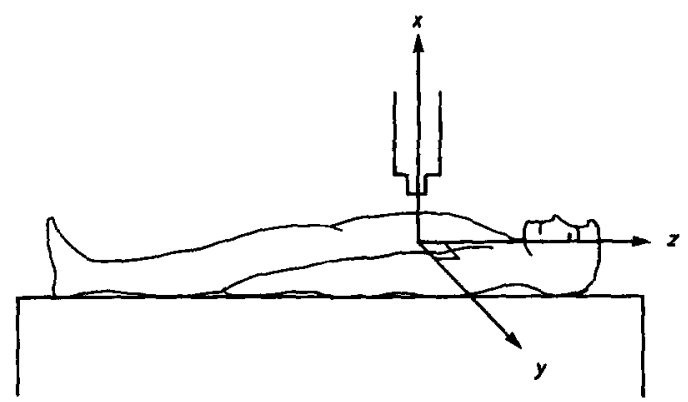

Fig. 2. - The co-ordinate system used. Note that the sign of the unit vector in the $x$-direction is opposite to the one used in most MCG measurements.

( ${ }^{()}$A. VAN OOSTerom and R. TH. VAN DaM: Adv. Oardiol., 16, 27 (1976).

(10) B. L. Carter, J. Morehead, M. S. Wolpert, S. B. Hammerschtag and H. J. GRIFFITHS: Cross-Sectional Anatomy, Computed Tomography and Oltrasound Correlation (New York, N. Y., 1977).

(11) R. C. BARR, M. RAMSEY, M. S. SPACH: IEEE Trans. Biomed. Eng., BME-24, 1 (1977).

(12) A. van Oosterom: Comput. J., 21, 253 (1978). 
system. The co-ordinate system used is given in fig. 2. An impression of the volume conductor can be obtained from fig. 3. A numerical approximation to eq. (3) was solved iteratively after deflation of those eigenvalues of the system of linear equations which are known a priori $\left({ }^{13}\right)$.

a)
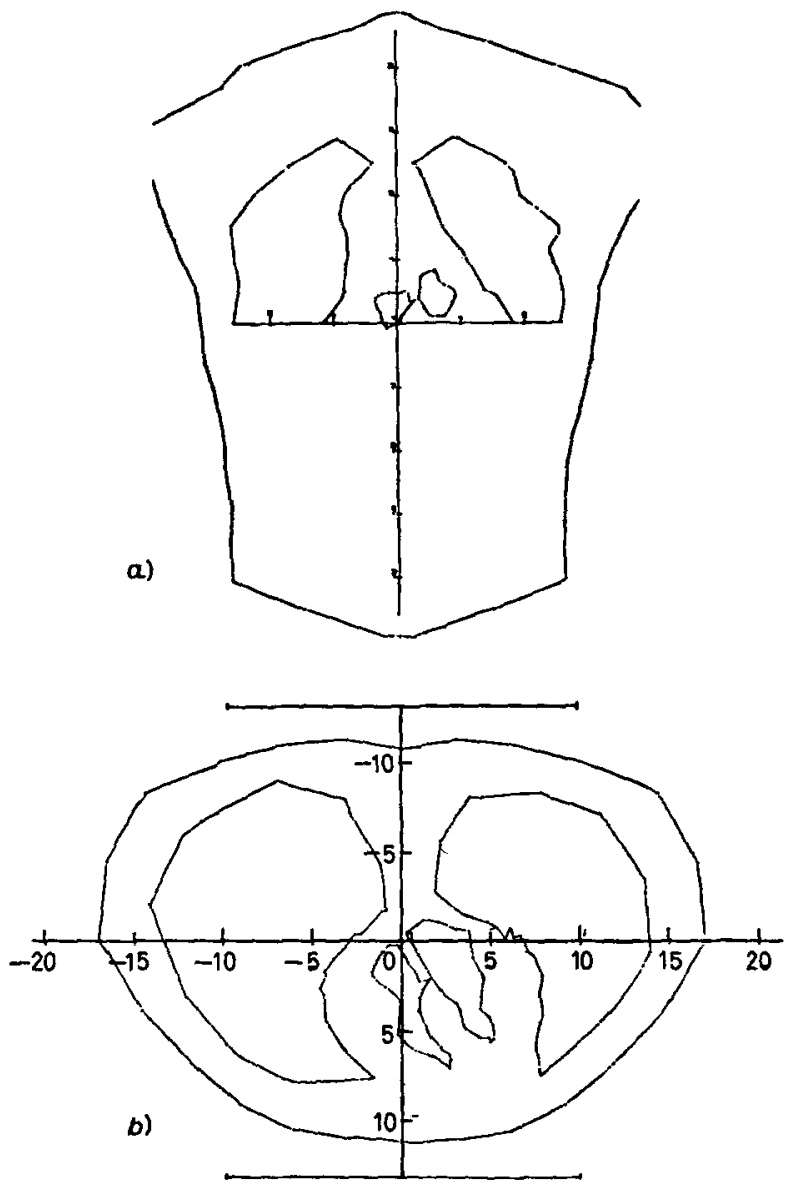

Fig. 3. - a) Frontal cross-section of the torso model at $x=2 \mathrm{em}$. b) Transverse crosssection of the torso model at $z=2 \mathrm{~cm}$.

With the potentials at each of the 574 vertex points known, a numerical approximation to eq. (1) was computed. The integral over one triangle as given in the last term of eq. (2) was approximated by

$$
\int_{\Delta} V\left(\boldsymbol{r}^{\prime}\right) \frac{\boldsymbol{r}-\boldsymbol{r}^{\prime}}{\left|\boldsymbol{r}-\boldsymbol{r}^{\prime}\right|^{3}} \times d \boldsymbol{S}^{\prime}=\frac{1}{3}\left(V_{1} \operatorname{tg} \alpha_{1}+V_{2} \operatorname{tg} \alpha_{2}+V_{3} \operatorname{tg} \alpha_{3}\right) \Omega \boldsymbol{e}_{m},
$$

(13) M. S. LYNN and W. P. TIMLAKE: SIAM J. Numer. Anal., 5, 303 (1968). 
where

$V_{1}$ is the potential in vertex 1 of this triangle, idem $V_{2}$ and $V_{3}$;

$\alpha_{1}$ is the angle between $r-r_{1}^{\prime}$ and the normal of the triangle with $r_{1}$ the co-ordinates of vertex 1 , idem $\alpha_{2}$ and $\alpha_{3}$;

$\boldsymbol{e}_{m}$ is the unit vector pointing in the direction of $\left(\boldsymbol{r}-\boldsymbol{r}^{\prime}\right) \times \mathrm{d} \boldsymbol{S}^{\prime}$ with $\boldsymbol{r}^{\prime}$ at the centre of the triangle, and

$\Omega \quad$ is the solid angle subtended by the triangle at the observation point.

The solid angle of a plane triangle has to be computed numerous times in both the numerical computation of the potential and that of the resulting magnetic field. For this we used a new algorithm which has recently been put forward $\left({ }^{14}\right)$.

\section{4. - Results.}

Several results of the computations described will be presented. The format used will be mainly that of isofunction lines of the magnetic-field component in the plane considered. The plane is parallel to the frontal plane of the subject. The computations were performed for the $x$-component of the magnetic field, $B_{x}$, over a $20 \mathrm{~cm}$ square with its centre point over the point $(0,0,0)$ (see fig. $3 b$ )). In the isofunction plots positive values are indicated by solid lines, negative values by dashed lines and zero is indicated by alternating dots and dashes. The difference between successive lines (i.e. delta) is given in the figure captions.

a) The magnetie field. In fig. 4 the square is $2 \mathrm{~cm}$ from the sternum, in the plane $x=14 \mathrm{~cm}$ as marked in fig. $3 b$ ). The map in fig. $4 a$ ) gives $B_{x}$ computed from the primary sources only. The map in fig. $4 b$ ) is generated by both primary and secondary sources by using the complete model (i.e. including lungs and cavities).

In fig. 5 the plane of observation is moved to the back at $2 \mathrm{~cm}$ from the spine, in the plane $x=-14 \mathrm{~cm}$ (see fig. 3b)). Figure 5a) is generated by the primary sources only, fig. 5b) by both primary and secondary sources. In this posterior view the direction of the $y$-axis is reversed in order to facilitate the comparison of measurements and computations.

(14) A. van OOSTERoM and J. StrackeE: The solid angle of a plane triongle, accepted for publication in IEEE Trans. Biomed. Eng. 

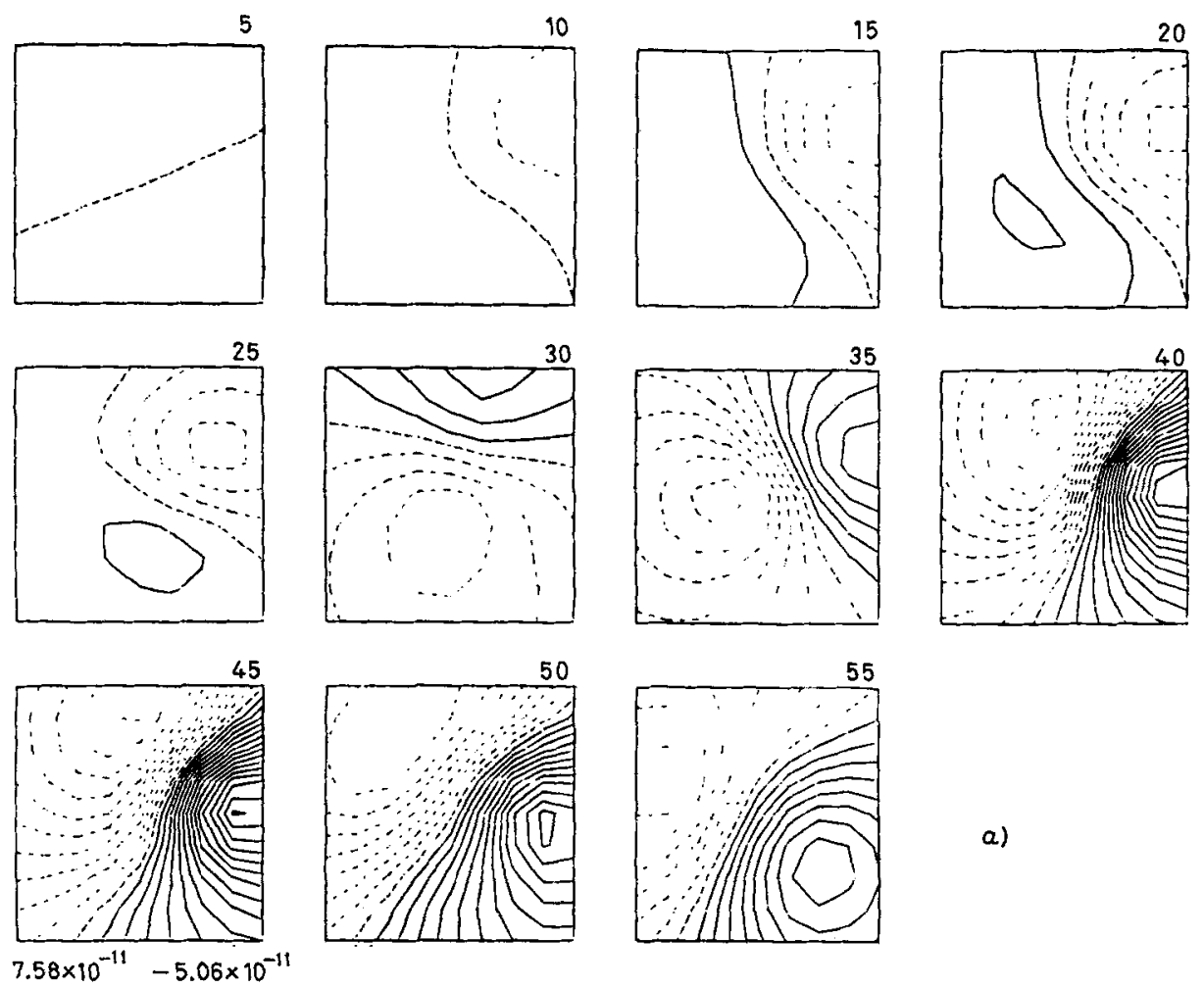

a)
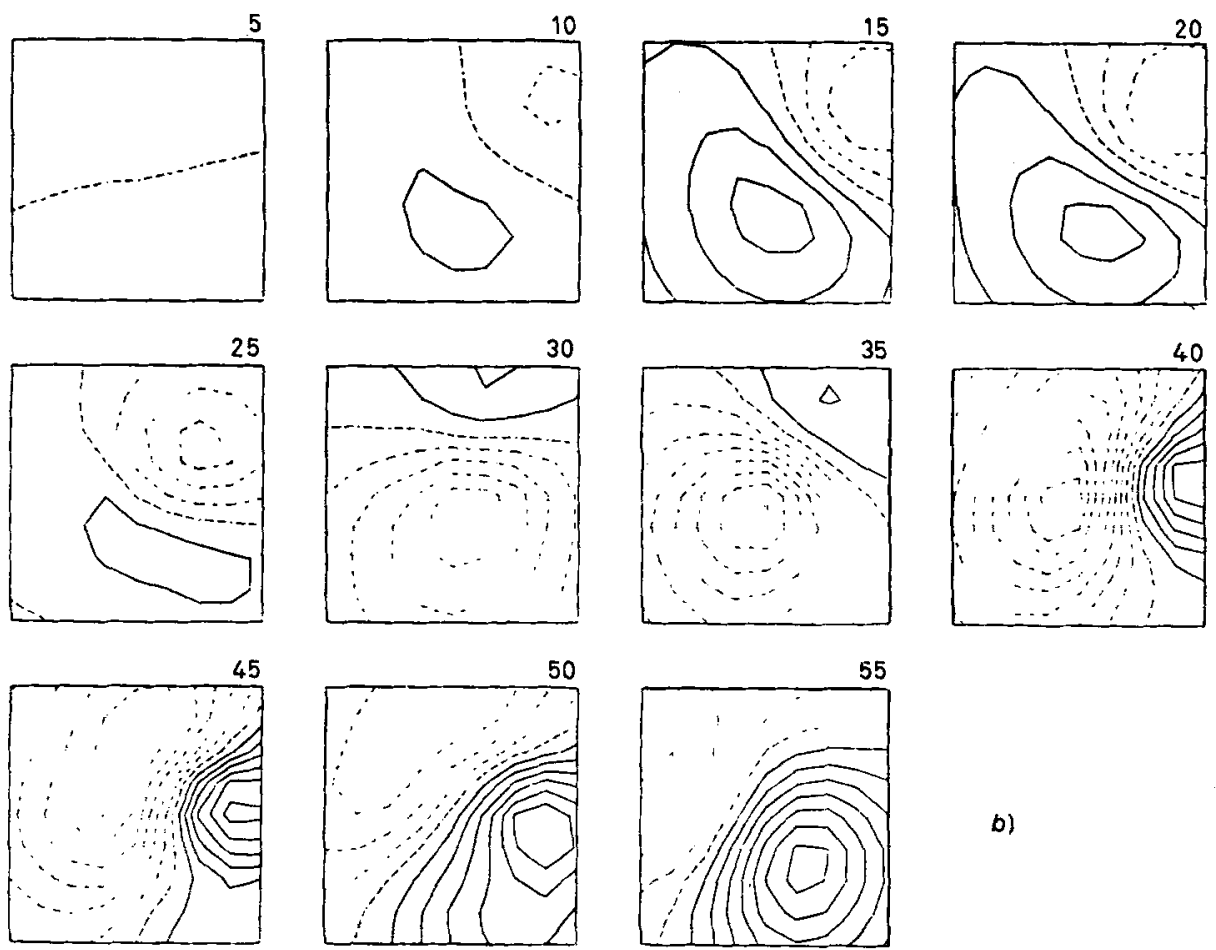

\section{b)}

Fig. 4. - Computed isomagnetic-field component maps in the plane $x=14 \mathrm{~cm}$. a) $B_{x}$ generated by the primary sources only. b) $B_{x}$ generated by both primary and secondary sources. Delta $=5 \cdot 10^{-12} \mathrm{~T}$. 

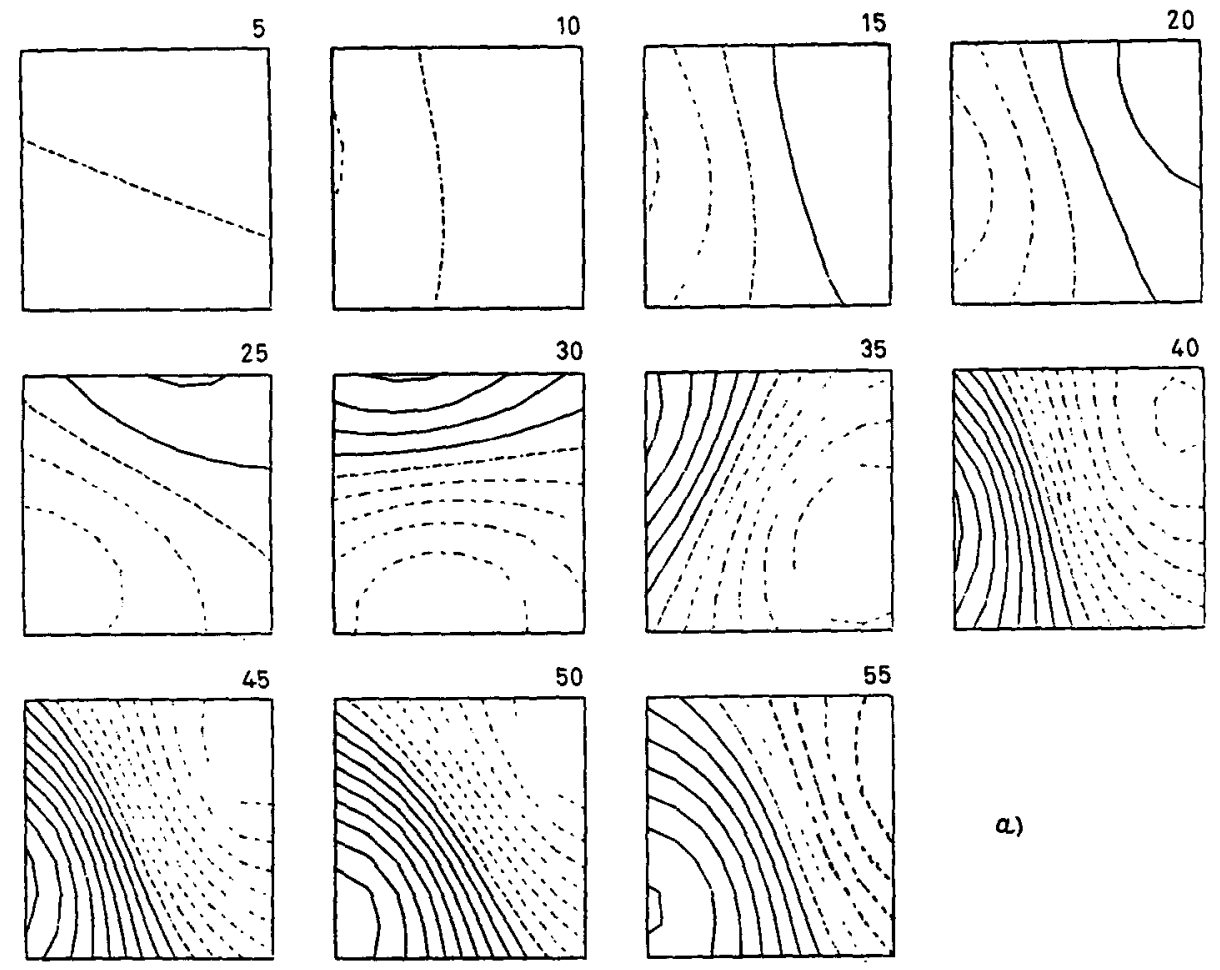

a)
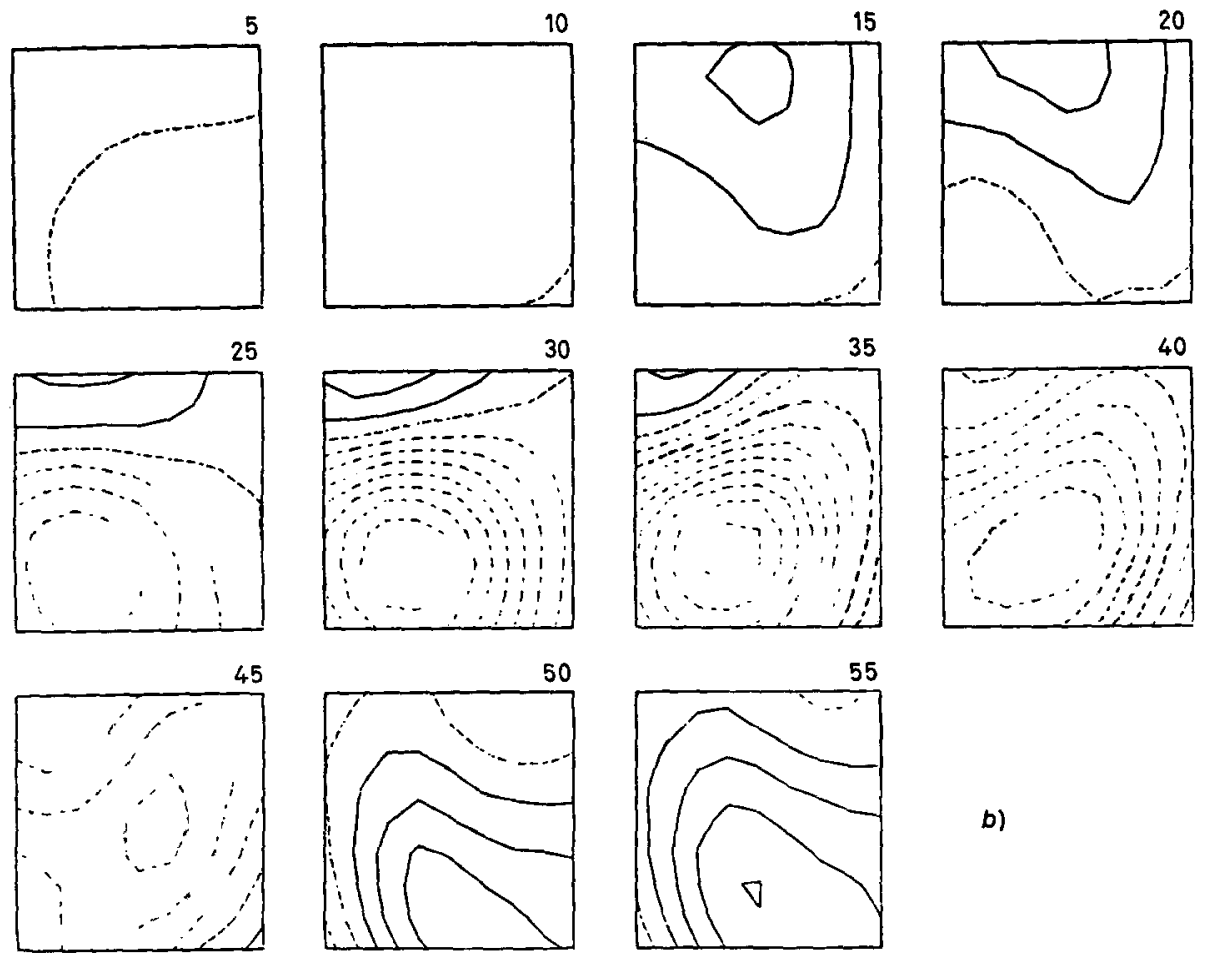

b)

Fig. 5. - Isomagnetic-field component maps in the plane $x=-14 \mathrm{~cm}$ (back). a) $B_{x}$ generated by the primary sources only. b) $B_{x}$ generated by both primary and secondary sources. Delta $=2 \cdot 10^{-12} \mathrm{~T}$. 

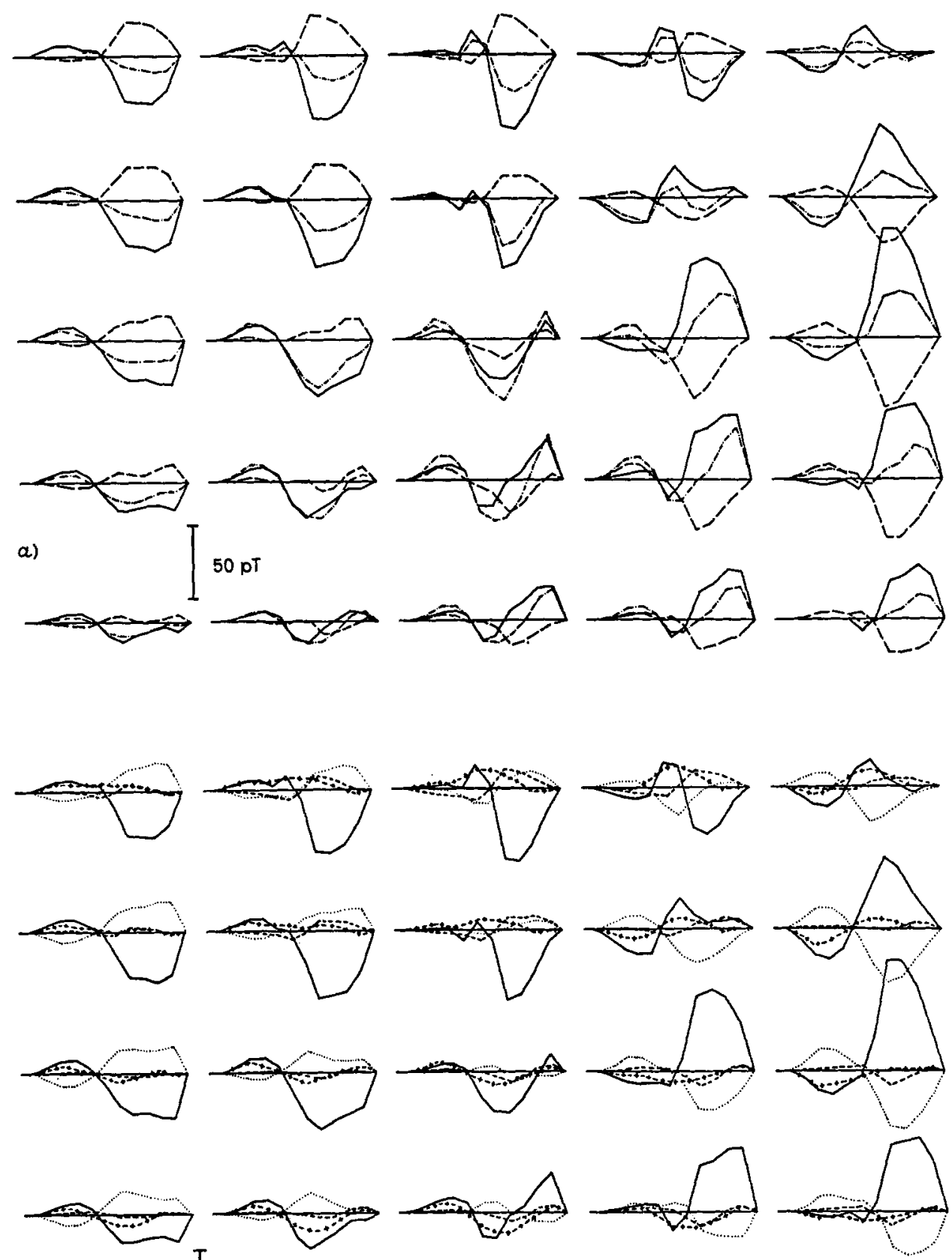

b)
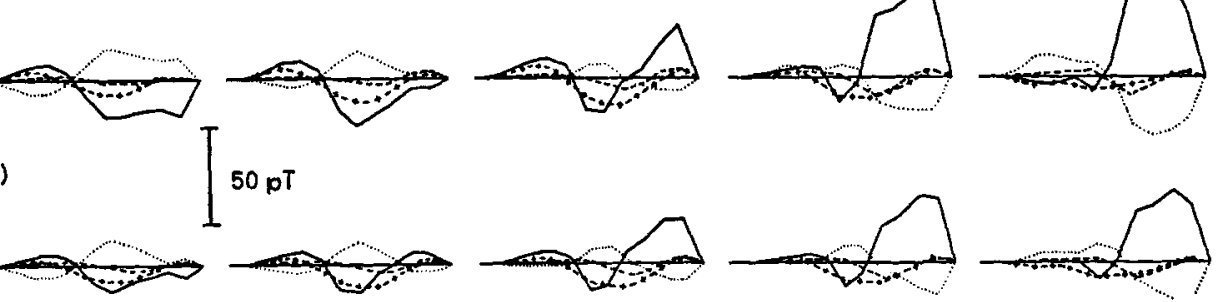

Fig. 6. - Computed MCGs during ventricular depolarization in the plane $x=14 \mathrm{~cm}$ for a model including lungs, cavities and torso boundary. The centre point of the grid is over the xiphoid. a) ___ primary sources only, _- _ all secondary sourees, -...- both primary and secondary sources. b) The contribution of the secondary sources separated into contributions from the cavities, the lungs and the torso surface. - primary source only, $\cdots$ torso surface, $--\ldots$ lungs, -+-+ - cavities. 
In fig. $6 a$ ) and $b$ ) the computed results are presented in the form of MCGs: the $x$-component of the magnetic field during ventricular depolarization is given as a function of time in the plane $x=14 \mathrm{~cm}$, in points $5 \mathrm{~cm}$ apart. The separate contributions due to the different boundaries are displayed in fig. 6b). In computing these separate contributions the secondary sources on all interfaces were present. The total contribution of all secondary sources is the summation of the separate ones.

In addition we studied isolated inhomogeneities in a bounded volume conductor. We found that the contribution to the magnetic field of secondary sources on the torso boundary tends to be reinforced by the presence of secondary sources on the lungs and cavities. The contribution of secondary sources on the lungs or on the cavities was reinforced or weakened by the presence of secondary sources on the cavities and on the lungs, depending on the time instant within the excitation cycle. The influence of interactions between the various torso compartments turns out to be small. A representative example is given in fig. 7, where the contribution of secondary sources on the torso boundary is given for four models, all at $40 \mathrm{~ms}$ after onset of ventricular depolarization. In fig. $7 a$ ) the torso is a homogeneous bounded conductor, in $7 b$ ) just the lungs, in $7 c$ ) only the intracavitary blood, in $7 d$ ) both intracavitary blood and lungs are present in the bounded torso.

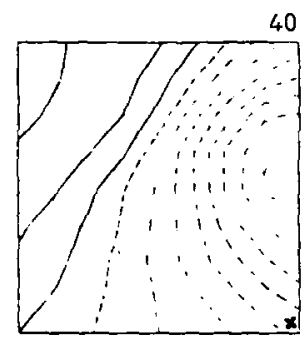

a)

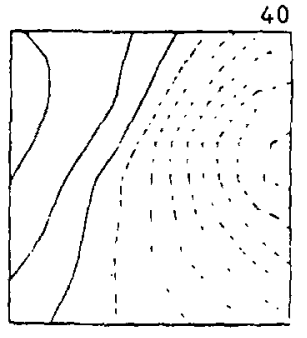

b)

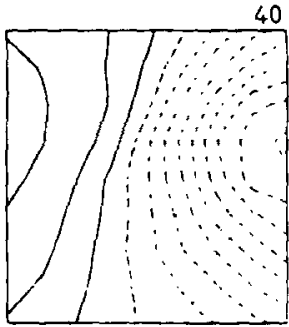

c)

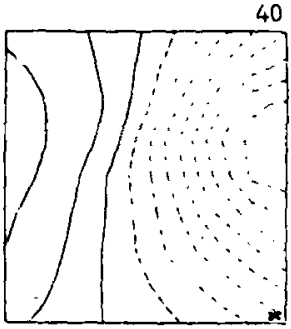

d)

Fig. 7. - Isomagnetic-field component maps in the plane $x=14 \mathrm{~cm}$ generated by the secondary sources on the torso boundary only by using 4 different models of the torso as mentioned in the text; delta in $5 \cdot 10^{-12} \mathrm{~T}$. The chosen time instant is $40 \mathrm{~ms}$.

b) The gradiometer. The flux through a flux transformer depends on the position of all sources relative to the pick-up coil. In order to estimate how accurately measured data represent the actual $x$-component of the magnetic field, we computed the total flux through a symmetric gradiometer. The gradiometer consisted of 3 parallel loops with 1,2 and 1 turn(s), respectively. The loop with 2 turns was wound in the opposite direction to the other ones. The distance between the loops (i.e. the baseline) was $6 \mathrm{~cm}$ along the $x$-axis and the radii of the loops were $1.5 \mathrm{~cm}$. In fig. $8 B_{\text {off }}$, which is defined as the total 

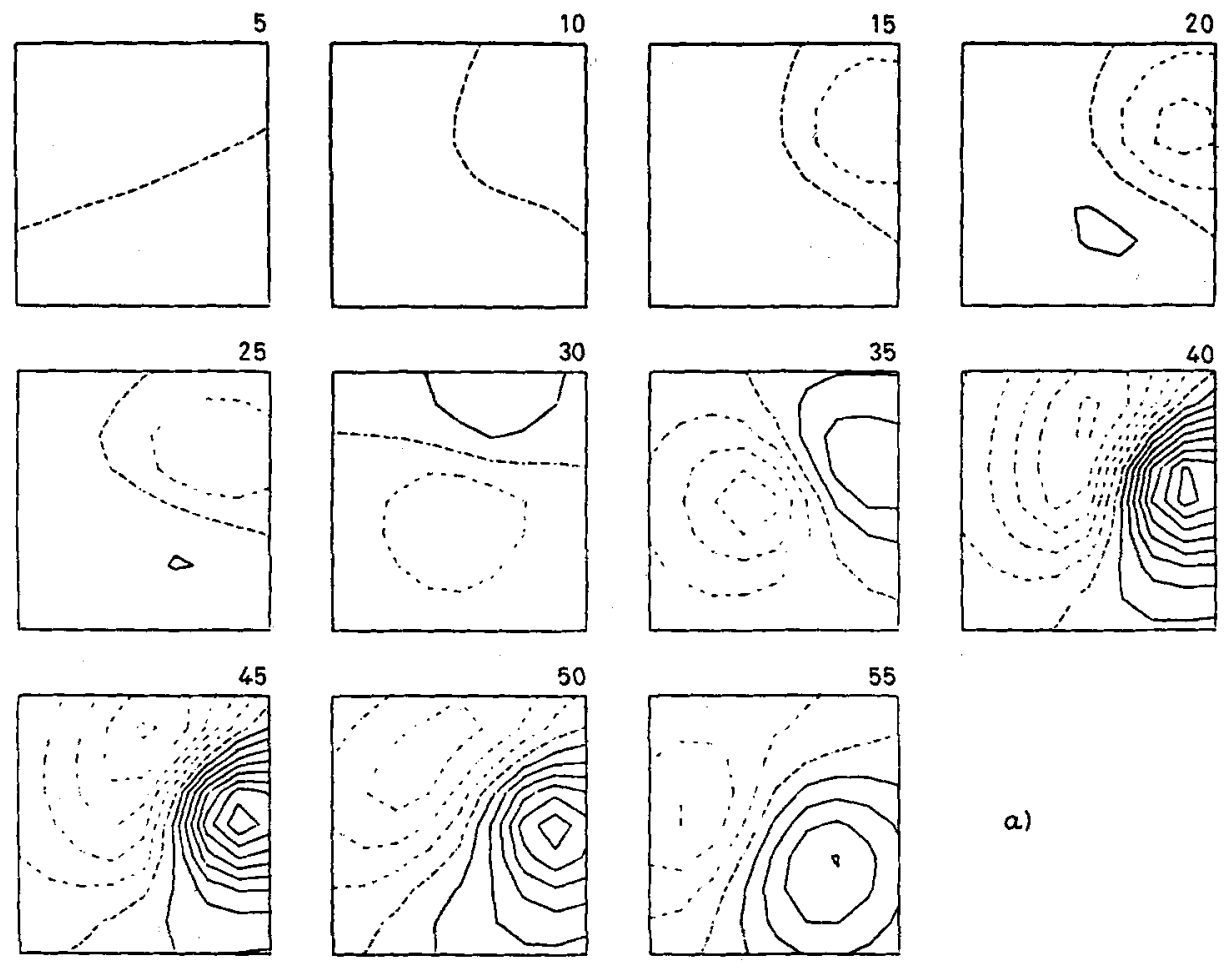

a)
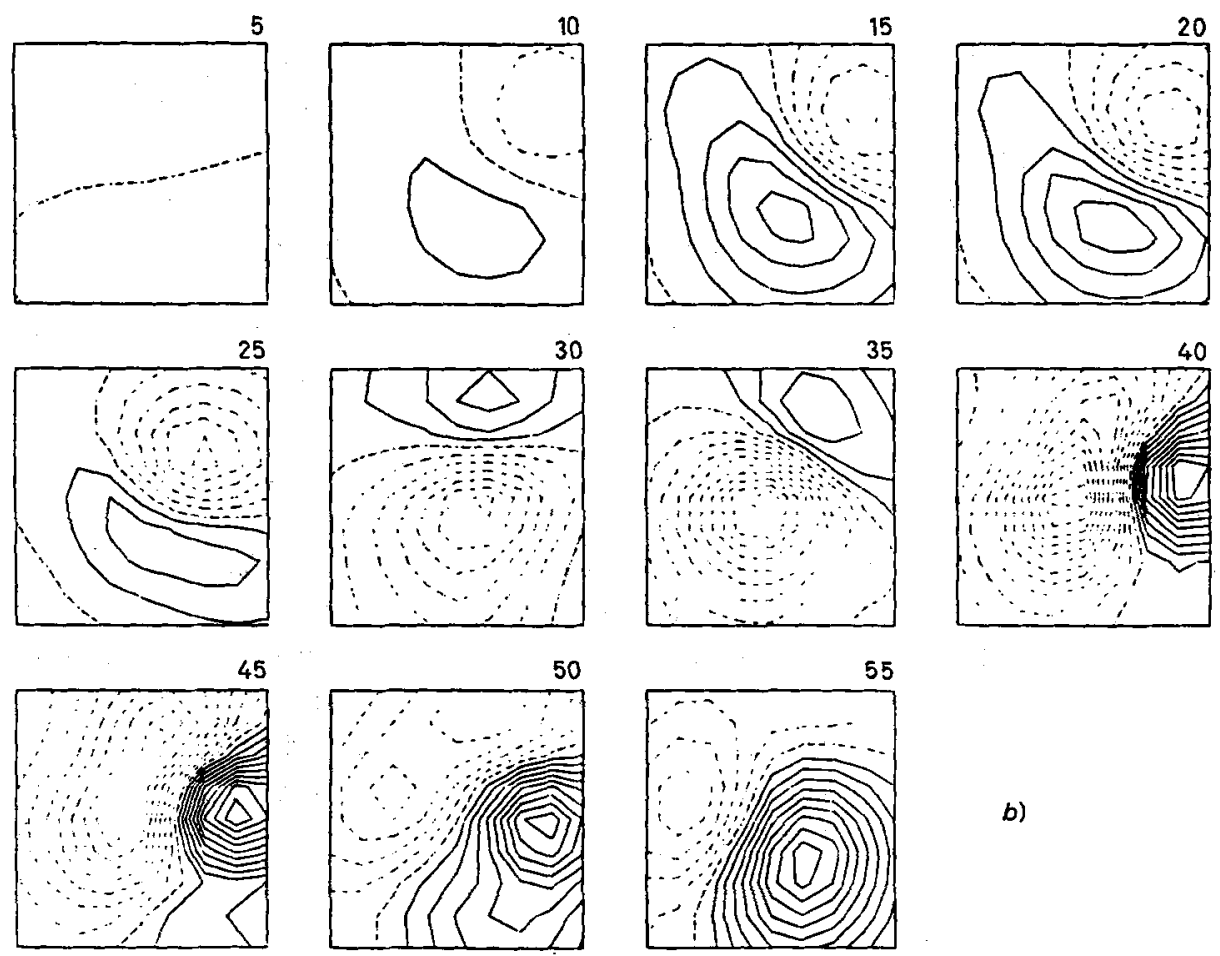

b)

Fig. 8. - Values of $B_{\text {eff }}$ in the plane $x=14 \mathrm{~cm}$ (gradiometer). a) $B_{\text {eff }}$ generated by the primary sources only. b) $B_{\text {eft }}$ generated by both primary and secondary sources. Delta $=2 \cdot 10^{-12} \mathrm{~T}$. 
flux through this gradiometer divided by the area of the pick-up loop, is mapped in the plane $x=14 \mathrm{~cm}$ (i.e. the plane where the pick-up loop is situated). The data in fig. $8 a$ ) are generated by the primary sources only and those of fig. $8 b$ ) by both primary and secondary sources.

\section{5. - Discussion.}

The results show that, in considering the magnetic field associated with cardiac activity, the contributions of the sum of all secondary sources involved counteract those of the primary sources. Although the general features of the maps are unchanged, large differences in particular values of $B_{x}$ are present. In this respect we confirm the findings of Cuffin $\left({ }^{8}\right)$.

The secondary sources on the torso boundary give a large contribution to the magnetic field, but do not change the general features appreciably, because the lines indicating the zero value almost coincide with those in corresponding maps generated by the primary sources only. In spite of the fact that the patterns and lines indicating zero value in the maps generated by secondary sources on the right and left lung and on the right and left cavity have little resemblance to the corresponding ones generated by primary sources, they do not change the general features, because the magnitude of their contributions is small.

Several computer model studies on MCGs have been reported previously (2,15-17). HORACEK modelled the ventricular activity using 1767 current dipoles with a fixed location and orientation but varying amplitudes, based on Durrer's data. His results are in fair agreement with ours, if we keep in mind that his simulated MCGs were based on the normal component in 36 points directly on the torso boundary, whereas we studied the $x$-component on a plane $2 \mathrm{~cm}$ from this boundary, this being a situation which can be realized experimentally. The influence of the blood mass in the heart is smaller in our study than that reported by HoRACEK; our data show that this contribution is more in proportion to the contribution of the primary sources. The comparison with Cuffin's calculations is again difficult because of the different format in which his results are presented. MHLER and GEsflowitz based their heart model also on Durrer's measurements, but adjusted the precise timing of the depolarization in the right ventricle with respect to that in the left ventricle and that in the interior wall with respect to that in the superior

(15) B. N. CUFFIN: Model studies of electrocardiograms and magnetocardiograms using realistic torso boundaries, Thesis, the Pennsylvania State University (1974).

(16) D. B. Geselowitz: IEEE Trans. Magn., MAG-16, 5 (1980).

(17) W. T. Miller and D. B. Geselowitz: Oirc. Re8., 43, 2 (1978). 
wall until the resulting dipole fell within normal vector cardiographic limits. They only took the secondary sources on the torso surface into account. Their results seem in fair agreement with both the measured isopotential maps and MCGs for normal human subjects $\left({ }^{18,19}\right)$. Our results show too large amplitudes during the later part of the depolarization cycle in comparison to measured isopotential maps and MCGs. We are aware of the fact that Durrer's data may not accurately represent what is occurring in the in situ human heart during the later part of the $Q R S$ cycle. The aim of our present study is to analyse the effect of inhomogeneities and not to simulate the most realistic ECGs and MCGs. Therefore, we decided not to take the liberty of changing the only measured data which are at one's disposal, but to use them as the best available basis.

RUDY and Plonsey ( ${ }^{20}$ ) demonstrated the importance of interactions between the various torso compartments in determining the potential distribution at the outer surface. It could be expected that these interactions are also important for the magnetic-field distribution outside the chest, because of the link between secondary sources and the potential distribution at each boundary. RUDY et al. concluded that the intracavitary blood contributed significantly to the enhancement of the peak potential on the outer surface; their conclusion was based on the results in certain positions on the surface. We do not find any such influence on the magnetic field. It is obvious that at any particular spot the influence may be quite large, whereas looking at the maps justifies the conclusion that the influence is rather small. Comparing, for instance, the point marked with a cross in fig. $7 a$ ) with the corresponding point in fig. 7d) we see in fig. $7 a) B_{x}=-16 \cdot 10^{-12} \mathrm{~T}$, while in $7 d$ ) $B_{x}=-9 \cdot 10^{-12} \mathrm{~T}$. Moreover, it should be pointed out that the models of the volume conductor and of the sources were different.

The ratio between the amplitudes at the back and front is high for the magnetic field in comparison to the equivalent ratio of the electric potential. This is confirmed by measurements $\left({ }^{18}\right)$. The magnetic field contributed by a source decreases rapidly in strength as a function of the distance from the point of observation. Near the chest the fields are predominantly generated by the sources of the anterior part of the heart and the fields at the back predominantly by the sources of the posterior part of the heart. This may provide a method to distinguish pathological heart conditions in the anterior section of the heart from those in the posterior section.

(18) M. Salrinen, P. Siltanen, P. J. Karp and T. E. Katila: Ann. Olin. Res., 10, 21 (1978).

(19) B. TAccardi, L. DE AMBroggi and C. ViganotTi: in The Theoretical Basis of Electrocardiology, edited by C. V. NeLson and D. B. Gesecowitz (Oxford, 1976), Chapt. 19.

(20) Y. RUdY and R. Plonsey: IEEE Trans. Biomed. Eng., BME-26, 7 (1979). 
The influence of the gradiometer is such that it slightly emphasizes the contribution of the primary sources relative to that of the secondary ones. This is to be expected because the secondary sources on the torso boundary and lungs nearest to the pick-up loop point perpendicularly to the loop (because parts of the surfaces of the torso and the lungs are almost parallel to the loops, see fig. $3 b)$ ), so they do not contribute to $B_{x}$. The secondary sources on the lung and the torso boundaries which do contribute are further removed from the pick-up loop than the nearest primary sources, their contribution decreases more than that of the primary sources, because the gradiometer is less sensitive to further-away sources than to nearby sources. The contribution of the secondary sources due to the cavities is decreased as much as that of the primary sources. A rough estimation of the ratios between $B_{\text {eff }}$ and $B_{x}$ gives

for both the secondary sources on the lungs and torso surface $B_{\text {etf }}=0.3 B_{x}$, for the primary sources $B_{\text {eff }}=0.5 B_{x}$,

for the secondary sources due to the cavities $B_{\text {eff }}=0.5 B_{x}$.

These ratios depend on the length of the baseline of the gradiometer. A suitable choice of the gradiometer baseline may help to minimize the influence of the volume conductor. This possibility has to be studied more extensively, as should be the influence of the enlargement of the diameters of the loops of the gradiometer. Maps of $B_{x}$ and maps of the flux through the pick-up loop divided by the area of the piek-up loop in the plane $x=14 \mathrm{~cm}$ are very similar. This means that measuring the flux by means of a loop with a diameter up till $3 \mathrm{~cm}$ is practically equivalent with measuring $B_{x}$ in the centre point of the loop. Maps of $B_{x}$ in the planes of the upper loops of the gradiometer appeared to be more dipolar than the map in the plane of the pick-up loop. This can be expected because the description of the entire heart activity by a single current dipole is a better approximation at a greater distance. This means that the general features of the maps generated by both primary and secondary sources are changed by the gradiometer.

In our study the current generators in the heart were described by uniform current dipole layers in spite of recent criticisms to the uniformity ${ }^{(21)}$ and the possibility that sources generating no electric field (vortex sources) make some contribution to the magnetic field (22).

The detailed cardiac and torso model described enables the simulation of heart diseases. Pathological heart conditions in either the anterior or the posterior division of the heart will be simulated in the near future. In this way we want to establish the possibility of recognizing the location of the

(21) D. E. Roberts, L. T. Hersch and A. M. Scher: Oirc. Res., 44, 5 (1979).

$\left.{ }^{22}\right)$ M. J. PETERS: The detection of bioelectric sources within the heart using a SQUID sensor, Thesis, Enschede, The Netherlands (1981). 
pathology from the magnetic-field distribution, especially in those cases in which a diagnosis based on ECG findings is difficult. Furthermore, the possibility that a gradiometer may help to focus on certain sources will be studied.

\section{- RIASSUNO(")}

Sono stati eseguiti calcoli numerici sul campo magnetico generato dal cuore durante la depolarizzazione ventricolare. Lo scopo di questo lavoro è studiare il contributo d'inomogeneità nel conduttore di volume al campo totale e stabilire l'influenza dei gradiometri usati nelle registrazioni sperimentali.

(*) Traduzione a cura della Redazione. 